\title{
Broadband superoscillation brings a wave into perfect three-dimensional focus
}

\author{
Alex M. H. Wong and George V. Eleftheriades* \\ The Edward S. Rogers Sr. Department of Electrical and Computer Engineering, University of Toronto, Toronto, Canada M5S 2E4
}

(Received 2 August 2016; revised manuscript received 6 January 2017; published 27 February 2017)

\begin{abstract}
The fundamental properties of a wave precludes it from being localized to subwavelength distances in all dimensions of the wave's existence. The inability to focus electromagnetic waves to an all-direction subwavelength spot limits the 3D resolution of a conventional imaging system to about half the imaging wavelength. A plethora of super-resolution imaging systems have been designed which obtain super-resolution in one or two (but not all) dimensions, but they suffer various restrictions in working distance and the classes of objects they can image. In this paper, we report a first investigation into a wave that is focused to subwavelength dimensions in all directions. After reviewing the physics of wave dispersion and diffraction which seemingly preclude this phenomenon, we sidestep these preclusions using a broadband superoscillation waveform and synthesize an all-direction subwavelength focus. We report the salient spatial and temporal features of this wave, and apply it to achieve $3 \mathrm{D}$ super-resolution imaging.
\end{abstract}

DOI: 10.1103/PhysRevB.95.075148

\section{INTRODUCTION}

Can one focus a waveform to subwavelength dimensions in all directions? The very nature of a wave seems to preclude its subwavelength localization in all its directions of existence. In the field of electromagnetics (the authors' primary interest), the inability to focus electromagnetic waves into a subwavelength spot leads to a fundamental resolution limit on classical imaging systems [1-3]. In many cases, one is especially interested in obtaining high resolution along one or two imaging dimensions within a 3D environment. Whereas it was long accepted that the diffraction limit precludes one from forming such images with a resolution beyond half the imaging wavelength, a plethora of super-resolution devices have been proposed, which sidestep the diffraction limit by involving and making clever use of evanescent waves [4-11]. These devices achieve super-resolution on the image plane by generating waveforms which decay exponentially in the longitudinal direction. Hence while these devices focus a waveform in $1 \mathrm{D}$ or $2 \mathrm{D}$, the waveform remains unfocused in the third (longitudinal) dimension; moreover, the rapid field decay along the longitudinal direction limits the working distances of these devices to about $1 / 4$ of the imaging wavelength. Alternatively, super-resolution microscopes are designed which exploit nonlinear optical effects or employ fluorescent biochemical labels [12-16], but their uses are limited to imaging prelabeled specimens or samples from selected material systems. A way to focus electromagnetic and acoustic waves to subwavelength dimensions in all directions would provide a direct path to super-resolution 3D imaging, with immediate applications in microscopy and many medical imaging modalities involving electromagnetic and ultrasound waves.

\footnotetext{
*gelefth@ece.utoronto.ca
}

Published by the American Physical Society under the terms of the Creative Commons Attribution 4.0 International license. Further distribution of this work must maintain attribution to the author(s) and the published article's title, journal citation, and DOI.
We think the curious phenomenon called superoscillation holds potential to focus a wave to subwavelength dimensions in all directions. Superoscillation refers to a waveform which, across a finite interval, oscillates quicker than the highest of its constituent frequency components [17-19]. Original studies on superoscillations dated at least from the 1960s, under the topic of prolate spheroidal wave functions [17]. The term superoscillation was coined in the 1990s by Berry [19] to describe a phenomenon observed by Aharonov and others on weak value measurement [18]. Almost immediately, it was realized that superoscillation applies to general waveform synthesis and analysis; this realization inspired immense interest in the study of superoscillations in various fields, which besides quantum mechanics also included mathematics, information theory, optics, and electromagnetics [20-34].

A curious and attractive feature of the superoscillation is that it expands the local effective bandwidth of a wave without involving evanescent waves and thereby enables the formation of subwavelength waveform features using only propagating waves. Previous works demonstrated the use of superoscillations to generate, far beyond the evanescent near field, subwavelength features on an image plane, which included hot spots [23-25], speckle patterns [26], and subdiffraction light beams [27]. These demonstrations led to various proposals for super-resolution far-field microscopes [28-34], which yielded subwavelength resolution in the transverse direction. However, whilst these works concentrated on achieveing super-resolution and subwavelength features on a transverse image plane (or a series of transverse image planes), the longitudinal wave profiles were either unexplored, mildly focused, or intentionally elongated.

This paper represents an investigation on superoscillationenabled all-direction subwavelength focusing and superresolution imaging. We begin by examining fundamental limitations due to the dispersion and diffraction of electromagnetic plane waves. We shall show that, while using evanescent waves, one is still bounded by the dispersion limitation; using superoscillations one may overcome both dispersion and diffraction limitations to achieve all-direction focusing beyond the diffraction limit. We then present a general design procedure for an all-direction subwavelength 
focused superoscillation waveform and discuss its salient spatial and temporal features. Finally, we demonstrate how an all-direction subwavelength-focused waveform may be applied to perform 3D super-resolution imaging.

\section{RESOLUTION LIMITS: DISPERSION AND DIFFRACTION}

For a single frequency waveform $u_{t}(\mathbf{r}, t)=u(\mathbf{r}) \exp (i \omega t)$ residing in a linear, homogeneous, and isotropic medium (such as free space), the source-free electromagnetic wave equation simplifies to the Helmholtz equation, which can be written in Cartesian coordinates as

$$
\left(\frac{\partial^{2}}{\partial q^{2}}+k_{q}^{2}\right) u(\mathbf{r})=0 \text { for } q=x, y, z .
$$

Here $k_{q}$ represents the $q$-directed spatial frequencies which are related through the dispersion relationship

$$
k_{m}^{2}=\left(\frac{\omega}{v_{m}}\right)^{2}=\left(\frac{\omega n}{c}\right)^{2}=k_{x}^{2}+k_{y}^{2}+k_{z}^{2},
$$

where $k_{m}$ is the spatial frequency (or the wavenumber), $v_{m}$ is the speed of light in the medium, $c$ is the speed of light in free space, and $n=\sqrt{\mu_{r} \epsilon_{r}}$ is the refractive index of the medium. Thus for a wave of a fixed frequency, $k_{m}$ is a constant which depends on the material's electromagnetic properties. The dispersion characteristic described by (2) regulates that (i) one cannot access waves with higher spatial frequency than $k_{m}$, which one typically needs for fast oscillation and hence high resolution; (ii) one also cannot access waves with lower spatial frequency than $k_{m}$, which means one lacks a bandwidth in the 3D $k$ space to form a complete basis, even after one accepts a resolution limit corresponding to $k_{m}$. Hence the dispersion of an electromagnetic wave in a material imposes fundamental limitations on the resolution of an arbitrary waveform in the medium.

We now steer our discussion towards a 2D $x y$ plane. We do this by considering the set of plane waves,

$$
u_{\mathrm{PL}}(\mathbf{r})=A_{\mathrm{PL}} \exp \left[-i\left(k_{x} x+k_{y} y+k_{z} z\right)\right],
$$

where $k_{x} / k_{m}$ and $k_{y} / k_{m} \in[-\infty, \infty]$. This set of plane waves forms a complete basis of the solution space of $u(\mathbf{r})$. Far away from material discontinuities the waveform can be described solely by propagating plane waves - the subset of (3) with

$$
k_{x}^{2}+k_{y}^{2} \leqslant k_{m}^{2}
$$

Through the mathematical uncertainty principle [35], the spectral width restriction (4) leads directly to a lower bound on the waveform's focal width on the $x y$ plane. This lower bound, known as Abbé's diffraction limit [1], is roughly equal to half the imaging wavelength.

Evanescent-wave-based imaging devices improve the imaging resolution by involving evanescent waves-plane waves with $\left|k_{x}\right|>k_{m}$ and/or $\left|k_{y}\right|>k_{m}$-in the process of image formation. This expands the waveform's bandwidth in the $\left(k_{x}, k_{y}\right)$ domain and hence improves an imaging device's resolution in the transverse direction. However, a quick substitution into (2) shows that this renders $k_{z}$ imaginary and inevitably leads to exponential waveform decay in the longitudinal direction. Devices such as the superlens [5] or the metascreen [10,11] may sidestep Abbé's diffraction limit and achieve super-resolution in at most two dimensions, but as the consequence of the dispersion relationship, they are fundamentally forbidden from achieving 3D super-resolution. (We refer the interested reader to a detailed discussion in Ref. [3].) Hence a fundamentally different paradigm is needed if one hopes to focus an electromagnetic wave to subwavelength dimensions in all directions.

Superoscillation-based imaging devices, on the other hand, do not suffer this fundamental limitation. This is because the subwavelength features in superoscillatory waves are accompanied by high-amplitude regions outside the superoscillation region [22]. This way, the generation of subwavelength spots does not amount to the subwavelength localization in the sense implied by the mathematical uncertainty principle. While the appearance of such high-amplitude regions can be seen as a drawback, previous works have shown that, for 1D and 2D superoscillations, high-amplitude regions can be adequately separated from the superoscillation region $[22,25,32,34,36-38]$ such that the superoscillation wave can be used to perform super-resolution imaging. In the following section we shall describe a method to construct a wave that is subwavelength focused in both transverse and longitudinal directions, hence leading to the demonstration of an alldirection subwavelength-focused wave.

\section{ALL-DIRECTION SUBWAVELENGTH FOCUSING}

To construct a 3D superoscillation we require a waveform which spans a finite volume in the 3D $k$ space. One would readily realize that this cannot be achieved with a single-frequency waveform, whose isofrequency contour forms a spherical shell in $k$ space as described by (2). Hence for our construction we use a broadband waveform with $N$ frequency components. Moreover, to enforce radial symmetry, we vary the spectral distribution only as a function of $k_{r}=|\mathbf{k}|$. Figure 1(a) shows a spectral diagram of the resultant composite waveform: it comprises $N$ spherical shells with radii $k_{r}=k_{n}=\omega_{n} / v_{m}$ (for $n=1$ to $N$ ), concentric to the origin. Mathematically, the composite waveform has the spectral distribution

$$
U(\mathbf{k}, t)=\sum_{n=1}^{N} A_{n}(t) \delta_{r}\left(k_{r}-k_{n}\right),
$$

and the corresponding spatial profile

$$
u(\mathbf{r}, t)=\sum_{n=1}^{N} A_{n}(t) j_{0}\left(k_{n} r\right)=\sum_{n=1}^{N} A_{n}(t) \frac{\sin k_{n} r}{k_{n} r},
$$

where $\left\{A_{n}(t)\right\}$ is a set of complex time-varying coefficients and $j_{0}(\cdot)$ is the zeroth order spherical Bessel function of the first kind. Tuning the weights $A_{n}(t)$ designs the waveform $u(\mathbf{r}, t)$. Since the resolution limitation for $u(\mathbf{r}, t)$ arises from the fastest varying component, we define $k_{m}=k_{N}$ to facilitate comparison to the diffraction limit.

We shall investigate the time-varying nature of the waveform in the following section. For the moment, we concentrate our attention at a point in time $t=t_{0}$, at which we design our waveform to form a 3D subwavelength focus. At this point in time the weights $\left\{A_{n}\left(t_{0}\right)\right\}$ can be treated as 


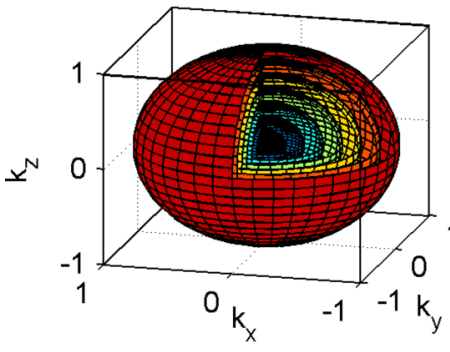

(a)

(c)

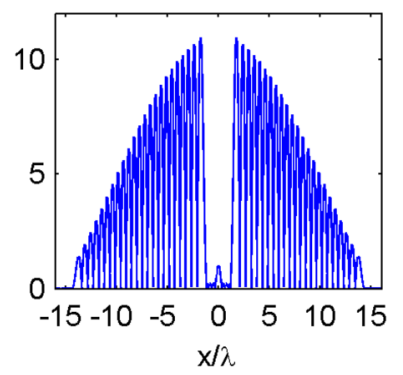

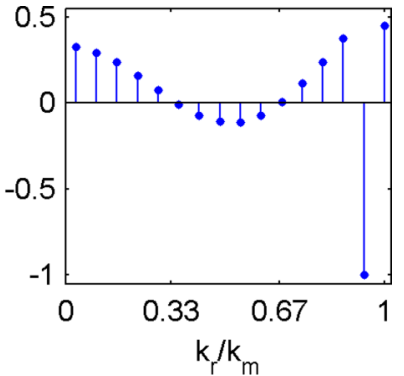

(b)

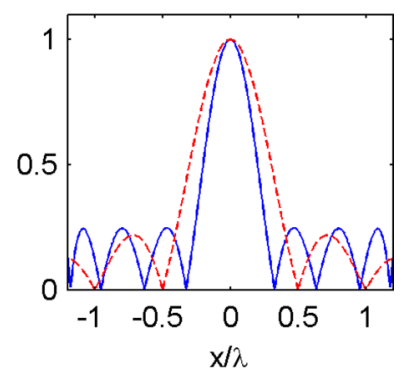

(d)
FIG. 1. 3D subwavelength focusing for a broadband waveform. (a) A diagram of spectral components involved. Each frequency component forms a spherical shell in $k$ space. (b) Weights $A_{n}$ for spherical Bessel functions (zeroth order, first kind) which would superimpose to form a 3D subwavelength focus. (c) The resultant electric field amplitude ( $x$-axis cut). The waveform is radially symmetric. (d) A closeup of the superoscillation region (blue, solid) plotted alongside the fastest varying spherical Bessel function $j_{0}\left(k_{m} r\right)$ (red, dashes). Subwavelength focusing to $66 \%$ of the diffraction limit is observed.

constants. We choose these constants using a method adapted from superdirective antenna array design [32,36,37,39]. With this method, we design a corresponding 1D superoscillation waveform through a Tschebyscheff polynomial matching procedure, then map the $1 \mathrm{D}$ waveform into $3 \mathrm{D}$ using a null matching procedure. The details of the mathematical formulation are provided in the Supplemental Material which accompanies this paper [40]. We choose for our construction 16 spherical Bessel functions with a linearly spaced set of wavenumbers $k_{n}=(n-1 / 2) \Delta k$ which correspond to the set of frequencies $w_{n}=v_{m} k_{n}$. Using our algorithm, we design a waveform with (i) minimal peak width, (ii) a superoscillatory region of $2.4 \lambda_{m}$, where $\lambda_{m}=2 \pi / k_{m}$ represents the wavelength of the highest frequency component, and (iii) a uniform sidelobe field level $\left(S L L_{i}\right)$ within the superoscillatory region which is below $25 \%$ that of the main peak (our algorithm would produce a waveform with a uniform sidelobe level $S L L_{f}$ which is somewhat lower than $\left.S L L_{i}\right)$.

Applying these parameters, we obtain the set of spherical Bessel function weights $\left\{A_{n}\left(t_{0}\right)\right\}$ shown in Fig. 1(b). Figure 1(c) shows a projection of the waveform along the $x$ axis. Figure 1(d) shows a closeup of this waveform in the superoscillatory region, alongside the fastest varying spherical Bessel function within this bandwidth: $u(r)=j_{0}\left(k_{m} r\right)$. While we only plot the $x$-axis profile, the waveform focus remains the same in other directions due to its radial symmetry. The full focal width at half maximum (FWHM) of the design waveform measures $0.40 \lambda_{m}$, which is $66 \%$ the width of the diffraction-limited 3D spot, whose FWHM is $0.603 \lambda_{m}$. Clearly, all-direction subwavelength focusing is achieved while (i) the sidelobes within the superoscillatory region remain comparable to the diffraction limit comparison, and (ii) the wave amplitude outside the superoscillation region remains reasonable-making this waveform amenable to robust synthesis. More aggressive superoscillation waves can be designed which feature narrower foci and lower sidelobe levels. In exchange, a higher proportion of the waveform energy would reside outside of the superoscillation region [22].

\section{TEMPORAL WAVEFORM PULSATION}

A salient feature of the all-direction subwavelength focus achieved in this manner is that it pulsates with the waveform's evolution in time. Since each constituent frequency component accumulates phase at a different temporal rate, the composite waveform varies in time with a temporal Bloch period $T_{B}=1 / \Delta f$, where $\Delta f$ is the frequency separation between adjacent spectral components. We describe this time evolution characteristic by explicitly writing $A_{n}(t)$ in (6) as a product of a complex constant with a temporal variation term. The resultant wave

$$
u_{t}(\mathbf{r}, t)=\sum_{n=1}^{N} A_{n} \exp \left(i \omega_{n} t\right) \frac{\sin k_{n} r}{k_{n} r}
$$

encapsulates both spatial and temporal variations of the waveform.

Figure 2(a) shows a time evolution of the wave profile around the time $t=0$, when the waveform attains the requisite phase alignment and achieves an all-direction subwavelength focus. Figure 2(b) plots the wave profile at a few selected points in time. As the waveform evolves from perfect focus $\left(t=0^{+}\right)$, we observe that the nearest sidelobes become more pronounced and eventually overwhelm the central peak. The central peak initially becomes narrower, but eventually widens to a level just above the diffraction limit, and remains that way throughout most of the temporal Bloch period. A blue dashed line in Fig. 2(a) outlines the $3 \mathrm{~dB}$ diameter of the central spot and shows the pulsation dynamics of the waveform's central peak. For this waveform, all-direction subwavelength focusing was achieved for $4.4 \%$ of the waveform period.

\section{3D SUPER-RESOLUTION IMAGING}

The pulsing nature of the 3D superoscillation focus can complicate its application to 3D imaging. This is because making a direct measurement of the subwavelength focus (or its scattering off an object) seemingly requires either (i) instrumentation which can capture the instantaneous (as opposed to time-averaged) waveform, or (ii) aptly designed time-gating schemes which filter out unwanted waveform portions. Whilst such developments represent promising directions for future research, in the remainder of this paper we show that the aforementioned conditions can be relaxed when one applies an all-direction subwavelength-focused waveform as a postprocessing filter. This greatly simplifies the required 


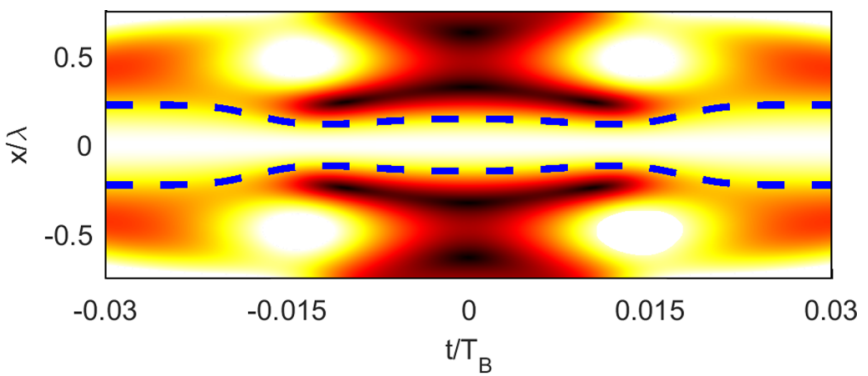

(a)

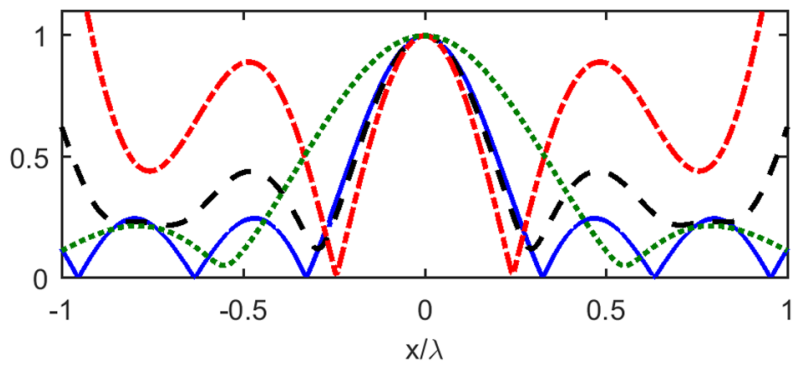

(b)

FIG. 2. Temporal waveform pulsation. (a) The time evolution of the waveform amplitude along the $x$ axis. The waveform is normalized to show constant strength at the principal peak at $x=0$. The blue dotted line denotes the $3 \mathrm{~dB}$ width of the $3 \mathrm{D}$ focal spot. (b) Waveform amplitude profiles at four different times: $t=t_{0}=0$ (blue, solid), $t=0.005 T_{B}$ (black, dash), $t=0.01 T_{B}$ (red, dash-dot), and $t=0.2 T_{B}$ (green, dotted). The spot width at $t=0.2 T_{B}$ is indicative of the waveform width for most of the period, when superoscillatory wave features are out of focus.

imaging apparatus and leads to a scheme readily applicable to existing imaging systems.

We show, through a numerical example, how an alldirection subwavelength focus may help one form a 3D super-resolution image. Figure 3(a) shows a geometric schematic with four point objects, arranged in tetrahedral formation and separated by distance $s$. The positions of the objects are $\mathrm{A}(0,-s / 2 \sqrt{6}, s / \sqrt{3}), \mathrm{B}(-s / 2,-$ $s / 2 \sqrt{6},-s / 2 \sqrt{3}), \mathrm{C}(s / 2,-s / 2 \sqrt{6},-s / 2 \sqrt{3})$, and $\mathrm{D}(0,-$

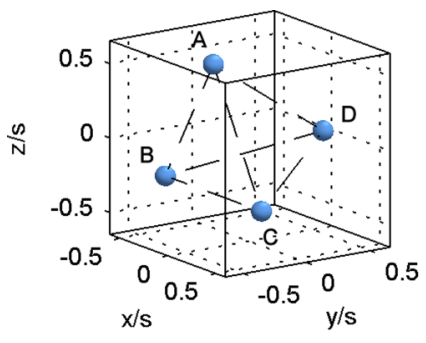

(a)

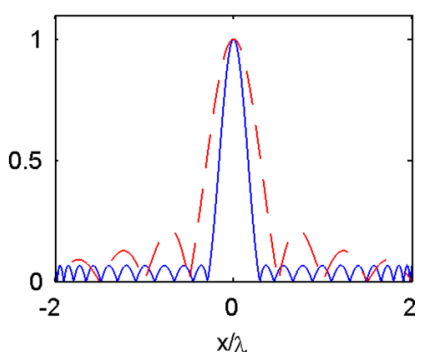

(b)
FIG. 3. Super-resolution imaging using an all-direction subwavelength focus. (a) A schematic showing the geometry of four point objects in the numerical experiment. The positions of the objects are $\mathrm{A}(0,-s / 2 \sqrt{6}, s / \sqrt{3}), \mathrm{B}(-s / 2,-s / 2 \sqrt{6},-s / 2 \sqrt{3}), \mathrm{C}(s / 2,-$ $s / 2 \sqrt{6},-s / 2 \sqrt{3})$, and $\mathrm{D}(0,-3 s / 2 \sqrt{6}, 0)$. (b) The superoscillation waveform used in the experiment, plotted with the spherical Bessel function $b_{0}(k r)$. Clearly, the superoscillation waveform contains an all-direction subwavelength focus and lower sidelobe levels.
$3 s / 2 \sqrt{6}, 0)$. A diffraction-limited 3D image of these objects can be obtained with a variety of methods and for electromagnetic waves ranging from rf to optical frequencies. As an example, Fig. 1 in the Supplemental Material [41] shows the apparatus for a microwave holographic method [42-44] for diffraction-limited 3D imaging. In this method, a broadband source-probe pair scans across a series of planes (with distances $D$ from the source plane). Inverse scattering is performed on the measured data to recover the object distribution. Under ideal circumstances in the measurement apparatus, this procedure recovers the object function with the limiting resolution of the 3D equivalent of Abbé's diffraction limit. Here we synthesize this ideal case by first generating a $3 \mathrm{D}$ object's spectral representation

$$
S_{\text {obj }}(\mathbf{k})=\sum_{s=1}^{4} \exp \left(-i \mathbf{k} \cdot \mathbf{r}_{s}\right),
$$

where $\mathbf{r}_{s}$ for $s=1$ to 4 represent the respective locations for objects A to D. Then we simulate diffraction effects by applying a 3D low-pass filter $F_{\mathrm{LP}}(\mathbf{k})$, which truncates the 3D spectrum to a sphere of radius $k_{m}$ :

$$
\begin{aligned}
& S_{\text {img }}(\mathbf{k})=S_{\text {obj }}(\mathbf{k}) F_{\mathrm{LP}}(\mathbf{k}), \quad \text { where } \\
& F_{\mathrm{LP}}(\mathbf{k})= \begin{cases}1, & k_{r} \leqslant k_{m}, \\
0, & \text { otherwise. }\end{cases}
\end{aligned}
$$

In general $S_{\mathrm{img}}(\mathbf{k})$ - the resolution-limited version of the object spectrum-is the Fourier transform of the object distribution as found from a diffraction-limited 3D imaging algorithm, such as the inverse scattering methods mentioned above. As such, it contains spectral information over the band of frequencies used to image the object. We plot the diffraction-limited object functions in two planes: (i) $y=$ $-s / 2 \sqrt{6}$, which intersects objects A, B, and C [Fig. 4(a)] and (ii) $x=0$, which intersects objects A and D [Fig. 4(c)]. For each plane we plot the object function for three separation distances: $s_{1}=\lambda_{m}$ (top row), $s_{2}=0.7 \lambda_{m}$ (middle row), and $s_{3}=0.4 \lambda_{m}$ (bottom row), where $\lambda_{m}=2 \pi / k_{m}$ represents the shortest wavelength of all waves involved. We observe that the objects, after passing through the low-pass filter representing the diffraction limit, are barely resolvable at $s_{1}$, unresolvable at $s_{2}$, and resembled a single point object at $s_{3}$. However, as we shall demonstrate, applying the filter corresponding to an all-direction subwavelength-focused wave resolves the objects at all these distances.

In this numerical example we use the all-direction subwavelength-focused waveform shown in Fig. 3(b), which features a focal width of $0.32 \lambda_{m}$ FWHM and a $4 \lambda_{m}$ superoscillation region within which the sidelobe level is $7.8 \%$ that of the main peak. As evident from the figure, the spot width and sidelobe level are significantly reduced compared to the spherical Bessel function of the highest frequency component. The design methodology for this waveform, as well as the design parameters used, are as explained in Sec. III. After we find the required weights $A_{n}$ for the aforementioned waveform, we substitute it into (5) to arrive at the waveform spectrum $U(\mathbf{k}, t)$ which we implement as a multiplicative spectral filter $S_{\mathrm{SO}}(\mathbf{k})$. Since $S_{\mathrm{SO}}(\mathbf{k})$ represents the spectral contents of (7) at the moment of optimal focus, its imaging performance stands 

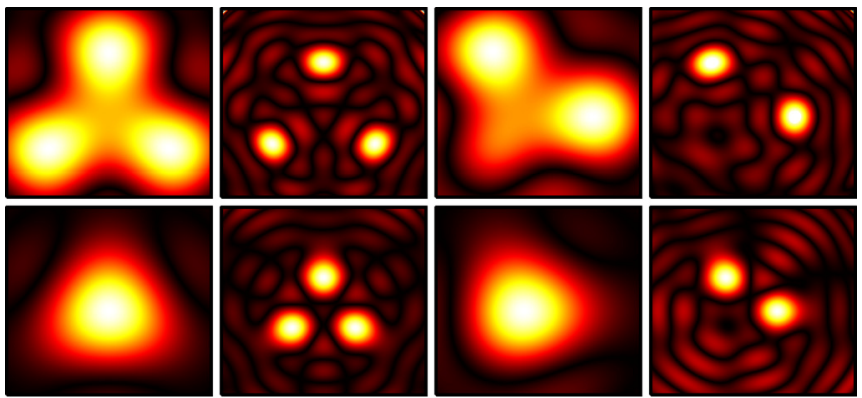

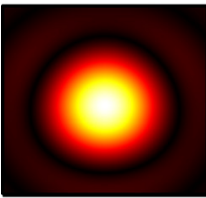

(a)

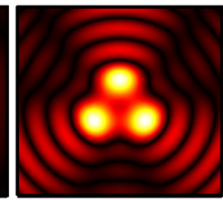

(b)

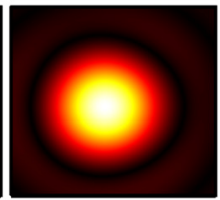

(c)

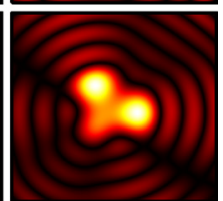

(d)
FIG. 4. Super-resolution imaging using an all-direction subwavelength focus. (a) Diffraction-limited images obtained for the cluster shown in Fig. 3(a), along the plane $y=-s / 2 \sqrt{6}$; (b) superresolution images equivalent of (a). (c) Diffraction-limited images obtained for the cluster shown in Fig. 3(a), along the plane $x=0$; (d) super-resolution images equivalent of (c). The separation distances are $s_{1}=\lambda_{m}$ (top row), $s_{2}=0.7 \lambda_{m}$ (middle row), and $s_{3}=0.4 \lambda_{m}$ (bottom row), where $\lambda_{m}=2 \pi / k_{m}$ represents the shortest wavelength involved in the imaging process.

unaffected by the waveform's pulsation in time. We use this spectral filter to reconstruct a super-resolution image:

$$
\begin{aligned}
S_{\text {recon }}(\mathbf{k}) & =S_{\text {img }}(\mathbf{k}) S_{\text {SO }}(\mathbf{k}), \\
S_{\text {recon }}(\mathbf{r}) & =\operatorname{iFT}\left\{S_{\text {recon }}(\mathbf{k})\right\},
\end{aligned}
$$

where $\operatorname{iFT}\{\cdot\}$ denotes the inverse Fourier transform operator.

Figures 4(b) and 4(d) display $s_{\text {recon }}(\mathbf{r})$ on the planes $y=$ $-s /(2 \sqrt{6})$ [Fig. 4(b)] and $x=0$ [Fig. 4(d)], again for distances $s_{1}=\lambda_{m}$ (top row), $s_{2}=0.7 \lambda_{m}$ (middle row), and $s_{3}=0.4 \lambda_{m}$ (bottom row). Evidently, all source objects are clearly resolved for all three distances. These figures show dramatic resolution improvement over the diffraction limit in all three directionsnot just over a 2D image area. We have therefore achieved 3D super-resolution imaging by numerically applying an alldirection subwavelength-focused wave.

\section{DISCUSSION}

In this section we make a few remarks regarding our demonstrations on superoscillation-based focusing and imaging.

\section{A. Synthesizing a 3D superoscillatory focus}

First we speculate how one might synthesize a 3D superoscillatory subwavelength focus. The designed subwavelength focus is composed solely of propagating waves. It can be synthesized by reproducing the corresponding electric and magnetic currents across any boundary that encloses the focus [45]. This alleviates the need for placing sources within subwavelength distance of the actual focus. The required electric and magnetic currents can be synthesized using a set of Huygens' metasurfaces - artificial surfaces with engineered electric and magnetic responses_- which surround the intended focus $[38,46,47]$. The generation of a 3D superoscillatory subwavelength focus would require synchronizing the phase and amplitude excitation to a large number of Huygens' sources. From a practical standpoint, this might be best facilitated by placing the metasurface on a spherical shell concentric to the focus, where geometric symmetry can be exploited to minimize the amount of tuning required of individual current sources, thus greatly simplifying the excitation network. Alternatively, a more traditional boundary (such as a rectangular box) can be taken, where one generates the appropriate surface currents on each wall using a combination of a simple source (such as a dipole) and a passive Huygens' metasurface [48].

\section{B. Sensitivity}

Next we remark on the sensitivity of the superoscillation waveforms, in regard to both the focusing and imaging simulations.

Figure 5(a) shows typical waveform variations when a random-phased additive white Gaussian noise is applied to the spherical Bessel function weights which constitute the 3D subwavelength focus shown in Fig. 1. This estimates sensitivity in forming an all-direction subwavelength focus when one deviates from the ideal weights due to practical imperfections. The signal to noise ratio is $15 \mathrm{~dB}$. As one can observe, the main lobe width remains unaffected amid slightly rising sidelobe levels. This shows the designed superoscillation waveform achieves subwavelength focusing with adequate robustness, which hence suggests it can be synthesized in an apparatus with reasonable precision.

We then investigate the sensitivity of the 3D superoscillation imaging process in a noisy environment. In this scenario, the precision of the superoscillation filter (i.e., the weighting of the spherical Bessel functions) does not pose a practical limit: the filter can attain utmost precision since it is numerically generated and applied. Instead, we suppose that due to noise or other limitations with the measurement apparatus errors are introduced into the object distribution $S_{\text {obj }}(\mathbf{r})$, within a spherical imaging region with a one-wavelength diameter (contributions outside this area can simply be truncated). This introduces a corresponding error in $S_{\text {obj }}(\mathbf{k})$ and ultimately affects the reconstructed image $s_{\text {recon }}(\mathbf{r})$ through (9) and (10). We model this error as a random phased, additive white Gaussian noise in the spatial domain. Figure 5(b) shows the $y$-plane and $x$-plane images for the case of $s=0.7 \lambda_{m}$, for SNRs of $10 \mathrm{~dB}, 5 \mathrm{~dB}$, and $2 \mathrm{~dB}$. From this subfigure, we see that the image quality remains reasonable even for the case of $\mathrm{SNR}=5$. For comparison, Fig. 5(c) shows the corresponding images before super-resolution reconstruction. This subfigure (i) demonstrates that substantial resolution improvement has been achieved even in the midst of noise and (ii) shows that the degradation shown in Fig. 5(b) does not come from the super-resolution algorithm, but instead also exists to a comparable degree in the original diffraction-limited image.

The sensitivity analyses in this section suggest superoscillation waveforms can be practically synthesized and used in superoscillation imaging systems. 


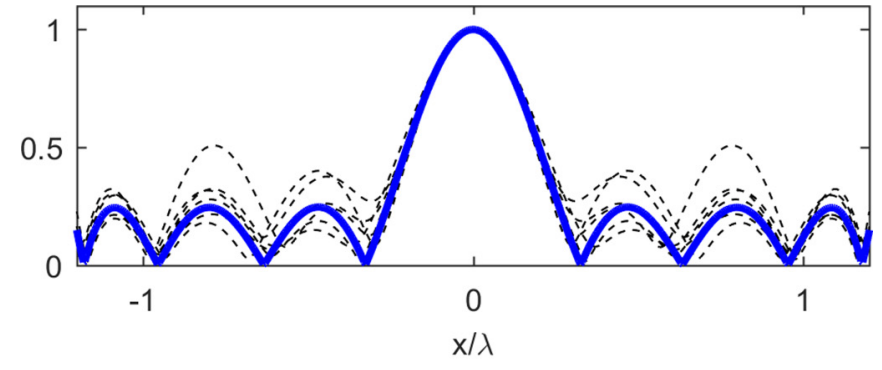

(a)
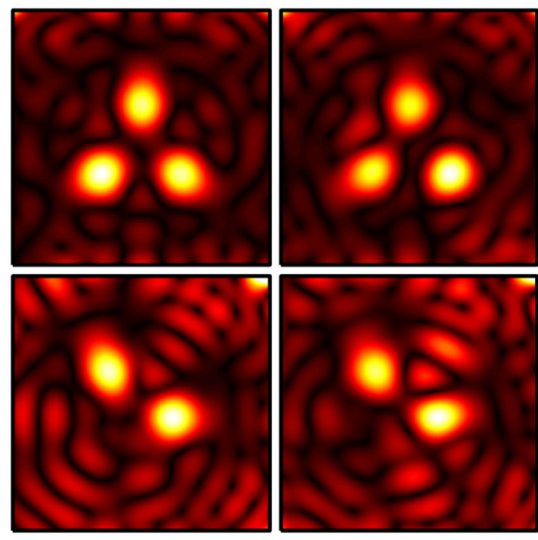

(b)
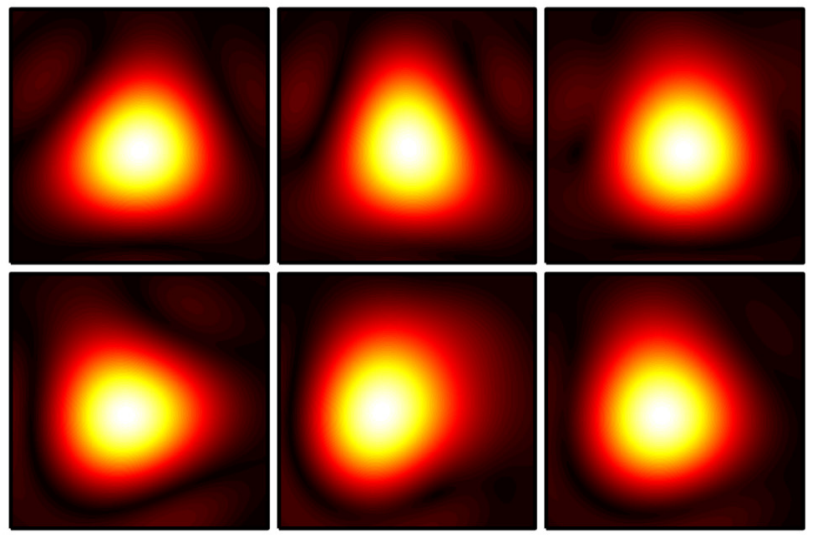

(c)

FIG. 5. Sensitivity analysis for superoscillation-based focusing and imaging. (a) Typical waveform variations (black, dashed) when a random-phased white Gaussian noise is added to the sub-wavelength focus shown in Fig. 1. Variations are shown for $\mathrm{SNR}=15 \mathrm{~dB}$. The original waveform is also shown for comparison (blue, solid). (b) $y$-plane (top-row) and $x$-plane (bottom-row) super-resolution images obtained in the presence of noise, with SNR $=10 \mathrm{~dB}$ (left), $5 \mathrm{~dB}$ (middle), and $2 \mathrm{~dB}$ (right). For this case the source separation is $s=0.7 \lambda_{m}$. (c) $y$-plane (top-row) and $x$-plane (bottom-row) diffraction-limited images in the presence of noise, with $\mathrm{SNR}=10$ $\mathrm{dB}$ (left), $5 \mathrm{~dB}$ (middle), and $2 \mathrm{~dB}$ (right). The source separation is the same as in (b).

\section{Optical super-resolution imaging}

The super-resolution imaging procedure we presented in the previous section applies in general, regardless of how an object distribution - a distribution of current density-is obtained. The procedure can be straightforwardly applied when an object distribution can be obtained from a procedure such as microwave holography, where one can measure the amplitude and phase of scattered waves from all angles and across the entire operation bandwidth. But what about imaging at optical frequencies, where it is nontrivial to measure the phase of an optical signal?

Measuring the phase of an optical signal is admittedly complicated, but very possible, using nonlinear techniques such as frequency-resolved optical gating (FROG) [49] or spectral phase interferometry for direct electric-field reconstruction (SPIDER) [50]. Alternatively, one can deduce the phase using linear techniques such as holographic interference with a reference wave. For situations when the phase of the optical signal cannot be measured, one can still solve an inverse problem to arrive at an approximation of the vectorial object distribution [51,52]. In such a case, it is conceivable that the phase retrieval step will cause some resolution degradation which would affect both the diffraction-limited object distribution and the super-resolution image in similar fashion. Notwithstanding, the improvement factor obtainable from the super-resolution imaging procedure hereby proposed should by and large remain unchanged.

\section{Comparison with 2D super-resolution schemes}

Next, we compare the 3D super-resolution imaging method demonstrated in this paper with a superoscillation-based 2D numerical super-resolution method, akin to the one proposed by Amineh et al. [44]. To obtain a comparable 2D numerical super-resolution result, we first design a $2 \mathrm{D}$ superoscillatory filter $S_{\text {SO-2D }}\left(k_{r}, k_{\phi}\right)$ with the same spot-width and sidelobe level as that for the 3D filter shown in Fig. 3(b). (We refer the interested reader to our earlier works [32,39] on 2D superoscillatory filter design.) Thereafter, we generate the 3D spectrum for diffraction-limited image $S_{\text {img }}(\mathbf{k})$ using (9), and reduce this to a $2 \mathrm{D}$ spectrum by performing an inverse Fourier transform in the direction normal to the $2 \mathrm{D}$ slice. The resultant 2D image $S_{\text {img.2D }}\left(k_{r}, k_{\phi}\right)$ is multiplied with the aforementioned 2D superoscillatory filter to obtain a reconstructed image along the plane of interest:

$$
\begin{aligned}
S_{\text {recon.2D }}(\mathbf{k}) & =S_{\text {img.2D }}\left(k_{r}, k_{\phi}\right) S_{\text {SO } \cdot 2 \mathrm{D}}\left(k_{r}, k_{\phi}\right), \\
S_{\text {recon.2D }}(\mathbf{r}) & =\operatorname{iFT}\left\{S_{\text {recon.2D }}\left(k_{r}, k_{\phi}\right)\right\} .
\end{aligned}
$$

Figure 6 compares the reconstructed images for the $3 \mathrm{D}$ and 2D super-resolution schemes for (i) a single point object, (ii) three point objects at locations $\mathrm{A}, \mathrm{B}$, and $\mathrm{C}$ with separation $s=0.7 \lambda$, and (iii) four point objects as depicted in Fig. 3(a). We examine the image along the plane $y=-s / 2 \sqrt{6}$, upon which the objects $\mathrm{A}, \mathrm{B}$, and $\mathrm{C}$ are colocated. The reconstructed image for the single point object verifies that we are comparing 2D and 3D images with a similar point-spread function. From the three-point and four-point reconstructions, we observe that, while the 2D super-resolution scheme succeeds in reconstructing a 2D scene in which all scatterers are colocated on the observation plane (which is the case for the three-point reconstruction), it fails when the scene also contains objects which lie away from the plane (four point reconstruction). Particularly, for the 2D super-resolution scheme, objects away from the target image plane are emphasized compared to objects lying on the plane. In general, linear electromagnetic 


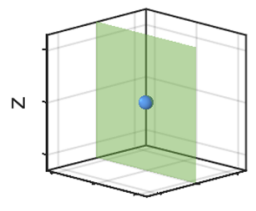

$\mathrm{x}$

y

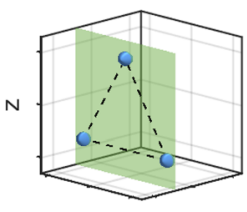

$x$

y

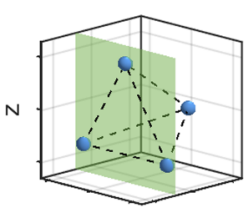

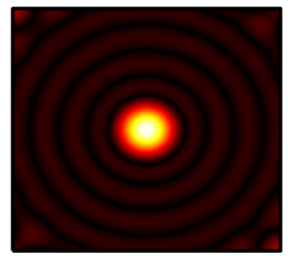
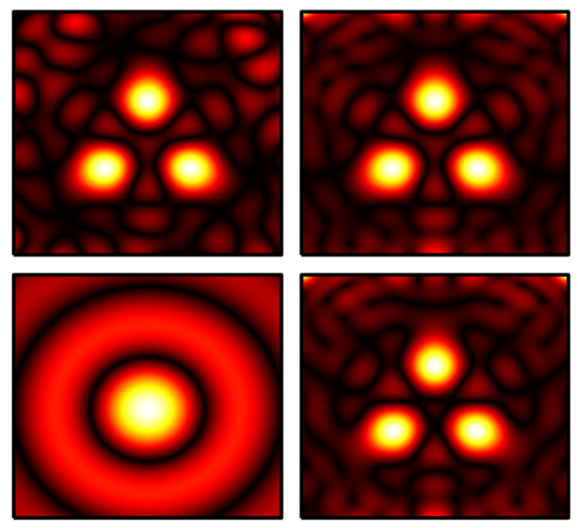

FIG. 6. Comparison between 3D and 2D super-resolution imaging. This figure shows imaging results for three different scenarios of single source (top row), three source on the same plane (middle row), and four sources as shown in Fig. 3(a). For the second and third cases the separation is $s=0.7 \lambda_{m}$; all images are taken along a constant- $y$ plane shown on the left panel. The middle column displays images processed by a 2D superoscillation filter; the right column displays images processed by a $3 \mathrm{D}$ superoscillation filter.

super-resolution schemes which provide 2D super-resolution do so at the expense of losing resolution in the third dimension. Hence the usage of 2D super-resolution techniques do not lead to 3D super-resolution, even when 2D image slices are taken at subwavelength increments. In contrast, the present work achieves 3D super-resolution by performing filter design with a full 3D consideration. Hence this work brings 3D super-resolution imaging to a level heretofore unreached by the application of 2D filters.

\section{E. Superoscillation imaging as a super-resolution method}

Finally, we aim to situate our work on 3D superoscillation imaging with respect to existing work on super-resolution methods. The usage of superoscillation to perform super- resolution 1D or 2D imaging has been previously known $[44,53,54]$; this work provides a way to obtain super-resolution simultaneously in all three dimensions. Fundamental limitations on certain features of a superoscillatory wave-namely the sharpness of the superoscillation, the duration of the superoscillation region, and the proportion of energy within the superoscillation region-have been established [22]. The effect of various kinds of noise has also been studied for a superoscillation imaging scheme [55]. Notwithstanding perceptions by some that superoscillation imaging schemes are too sensitive to be practical, in this work we demonstrated 3D subwavelength focused superoscillation waveforms with adequate robustness. In particular, in Sec. VIB we showed that one can robustly generate the proposed focus and use the proposed concept in a super-resolution imaging method. $3 \mathrm{D}$ superoscillation imaging is a very general super-resolution technique in that it does not assume a certain material system, requires no scanning and labeling, and does not rely on any prior knowledge about an object, other than the fact that it exists within a well defined image region. If prior information is available, that information can perhaps be included to improve the resolution [56] in conjunction to superoscillationbased super-resolution. Such composite imaging schemes form interesting directions of future research.

\section{CONCLUSION}

This work has reported the construction of an electromagnetic wave which is focused to a subwavelength spot in all directions. We clarified the effects of wave dispersion and diffraction which have heretofore precluded the conception and demonstration of a waveform focused to subwavelength dimensions in all directions. Thereafter we surpassed these limitations and constructed such a waveform as a broadband superoscillation wave. The all-direction subwavelength focus achieved a radially symmetric spot with its size reduced to $66 \%$ of the diffraction limit, while its sidelobe level (within the superoscillation region) remains unaffected. After studying the spatial and temporal features of this waveform, we applied it in a numerical fashion to achieve 3D super-resolution imaging, whereby we successfully reduced the minimal resolvable distance to about $40 \%$ of the diffraction limit. The ability to perform 3D subwavelength focusing and imaging should open new and attractive possibilities to wide-ranging fronts of high-resolution scientific and medical imaging.
[1] E. Abbé, Archiv. f. mickroskopische anat. 9, 413 (1873).

[2] L. Rayleigh, Philos. Mag. 42, 167 (1896).

[3] F. Mesa, M. J. Freire, R. Marqués, and J. D. Baena, Phys. Rev. B 72, 235117 (2005).

[4] D. W. Pohl, W. Denk, and M. Lanz, Appl. Phys. Lett. 44, 651 (1984).

[5] J. B. Pendry, Phys. Rev. Lett. 85, 3966 (2000).

[6] G. V. Eleftheriades, A. K. Iyer, and P. C. Kremer, IEEE Trans. Microwave Theory Tech. 50, 2702 (2002).

[7] N. Fang, H. Lee, C. Sun, and X. Zhang, Science 308, 534 (2005).
[8] Z. Jacob, L. V. Alekseyev, and E. Narimanov, Opt. Express 14, 8247 (2006).

[9] A. Salandrino and N. Engheta, Phys. Rev. B 74, 075103 (2006).

[10] L. Markley, A. M. H. Wong, Y. Wang, and G. V. Eleftheriades, Phys. Rev. Lett. 101, 113901 (2008).

[11] A. Grbic, L. Jiang, and R. Merlin, Science 320, 511 (2008).

[12] W. Denk, J. H. Strickler, and W. W. Webb, Science 248, 73 (1990).

[13] S. W. Hell and J. Wichmann, Opt. Lett. 19, 780 (1994).

[14] S. W. Hell and M. Kroug, Appl. Phys. B 60, 495 (1995). 
[15] M. J. Rust, M. Bates, and X. Zhuang, Nat. Methods 3, 793 (2006).

[16] E. Betzig, G. H. Patterson, R. Sougrat, O. W. Lindwasser, S. Olenych, J. S. Bonifacino, M. W. Davidson, J. LippincottSchwartz, and H. F. Hess, Science 313, 1642 (2006).

[17] D. Slepian and H. O. Pollak, Bell Labs Tech. J. 40, 43 (1961).

[18] Y. Aharonov, J. Anandan, S. Popescu, and L. Vaidman, Phys. Rev. Lett. 64, 2965 (1990).

[19] M. V. Berry, Faster than Fourier (World Scientific, Singapore, 1994), pp. 55-65.

[20] A. Kempf, J. Math. Phys. 41, 2360 (2000).

[21] M. Calder and A. Kempf, J. Math. Phys. 46, 012101 (2005).

[22] P. J. S. G. Ferreira and A. Kempf, IEEE Trans. Signal Process. 54, 3732 (2006).

[23] F. M. Huang, Y. Chen, F. J. Garcia de Abajo, and N. I. Zheludev, J. Opt. A: Pure Appl. Opt. 9, S285 (2007).

[24] M. Mazilu, J. Baumgartl, S. Kosmeier, and K. Dholakia, Opt. Express 19, 933 (2011).

[25] A. M. H. Wong and G. V. Eleftheriades, IEEE Trans. Antennas Propag. 59, 4766 (2011).

[26] M. R. Dennis, A. C. Hamilton, and J. Courtial, Opt. Lett. 33, 2976 (2008).

[27] K. G. Makris and D. Psaltis, Opt. Lett. 36, 4335 (2011).

[28] T. Brunet, J. L. Thomas, and R. Marchiano, Phys. Rev. Lett. 105, 034301 (2010).

[29] J. Baumgartl, S. Kosmeier, M. Mazilu, E. T. F. Rogers, N. I. Zheludev, and K. Dholakia, Appl. Phys. Lett. 98, 181109 (2011).

[30] S. Kosmeier, M. Mazilu, J. Baumgartl, and K. Dholakia, J. Opt. 13, 105707 (2011).

[31] E. T. F. Rogers, J. Lindberg, T. Roy, S. Savo, J. E. Chad, M. R. Dennis, and N. I. Zheludev, Nat. Mater. 11, 432 (2012).

[32] A. M. H. Wong and G. V. Eleftheriades, Sci. Rep. 3, 1715 (2013).

[33] E. T. F. Rogers, S. Savo, J. Lindberg, T. Roy, M. R. Dennis, and N. I. Zheludev, Appl. Phys. Lett. 102, 031108 (2013).

[34] D. Tang, C. Wang, Z. Zhao, Y. Wang, M. Pu, X. Li, P. Gao, and X. Luo, Laser Photon. Rev. 9, 713 (2015).

[35] G. B. Folland and A. Sitaram, J. Fourier Anal. Appl. 3, 207 (1997).

[36] A. M. H. Wong and G. V. Eleftheriades, IEEE Antennas Wireless Propag. Lett. 9, 315 (2010).

[37] A. M. H. Wong and G. V. Eleftheriades, IEEE Trans. Microwave Theory Tech. 59, 2173 (2011).
[38] A. M. H. Wong and G. V. Eleftheriades, Sci. Rep. 5, 8449 (2015).

[39] A. M. H. Wong and G. V. Eleftheriades, The 8th European Conference on Antennas and Propagation (EuCAP 2014) (IEEE, New York, 2014), p. 1340 .

[40] See Supplemental Material at http://link.aps.org/supplemental/ 10.1103/PhysRevB.95.075148 for the detailed mathematical formulation on $1 \mathrm{D}$ and $3 \mathrm{D}$ superoscillatory function design.

[41] See Supplemental Material at http://link.aps.org/supplemental/ 10.1103/PhysRevB.95.075148 for a diagram showing the apparatus for a microwave holographic method.

[42] R. K. Amineh, M. Ravan, A. Khalatpour, and N. K. Nikolova, IEEE Trans. Antennas Propag. 59, 4777 (2011).

[43] R. K. Amineh, A. Khalatpour, and N. K. Nikolova, IEEE Trans. Antennas Propag. 60, 3526 (2012).

[44] R. K. Amineh and G. V. Eleftheriades, Opt. Express 21, 8142 (2013).

[45] R. F. Harrington, in Time-harmonic Electromagnetic Fields, edited by D. G. Dudley, IEEE Pass Series on Electromagnetic Wave Theory (IEEE Press, New York, 2001).

[46] A. M. H. Wong and G. V. Eleftheriades, IEEE International Symposium on Antennas and Propagation (IEEE, New York, 2015).

[47] A. M. H. Wong and G. V. Eleftheriades, IEEE Mediterranean Electrotechnical Conference (MELECON) (IEEE, New York, 2016).

[48] A. Epstein, J. P. S. Wong, and G. V. Eleftheriades, Nat. Commun. 7, 10360 (2016).

[49] R. Trebino, K. W. DeLong, D. N. Fittinghoff, J. N. Sweetser, M. A. Krumbgel, B. A. Richman, and D. J. Kane, Rev. Sci. Instrum. 68, 3277 (1997).

[50] C. Iaconis and I. A. Walmsley, Opt. Lett. 23, 792 (1998).

[51] R. W. Gerchberg and W. O. Saxton, Optik 35, 237 (1972).

[52] J. R. Fienup, Appl. Opt. 21, 2758 (1982).

[53] C. W. Barnes, J. Opt. Soc. Am. 56, 575 (1966).

[54] K. Piché, J. Leach, A. S. Johnson, J. Z. Salvail, M. I. Kolobov, and R. W. Boyd, Opt. Express 20, 26424 (2012).

[55] C. K. Rushforth and R. W. Harris, J. Opt. Soc. Am. 58, 539 (1968).

[56] C. L. Byrne, R. M. Fitzgerald, M. A. Fiddy, T. J. Hall, and A. M. Darling, J. Opt. Soc. Am. 73, 1481 (1983). 